Hanno Pahl*

\title{
Steuerungsabstinenz als Ordnungsvision: Soziologische Beobachtungen zur Rational Expectations Revolution in der Makroökonomik
}

DOI 10.1515/zksp-2015-0014

Zusammenfassung: Die moderne Makroökonomik ist seitens der Soziologie nahezu unbeobachtet und unkommentiert geblieben. Dieses Versäumnis betrifft insbesondere die Arbeiten von Robert Lucas, die zwar außerhalb der Disziplin nicht gleichermaßen sichtbar waren wie die politisch wirkungsmächtigen Eingaben Friedmans und Hayeks, denen aber ein nicht zu unterschätzender Einfluss auf die Fortentwicklung der modernen Makroökonomik zugeschrieben werden muss (Rational Expectations Revolution, New Classical Macroeconomics). Auf indirektem Weg - wesentlich über die Propagierung neuer Modellierungstechniken und neuer wissenschaftlicher Standards - wurden eine dezidierte Steuerungsskepsis und eine marktaffirmative Weltsicht kultiviert. Der Text offeriert einen pointierten Überblick über makroökonomische Entwicklungen nach dem Ende der Hegemonie des Keynesianismus der neoklassischen Synthese und legt das Augenmerk einerseits auf Modellierungstechniken, andererseits auf den Wandel von Steuerungsvorstellungen. Es wird eine Perspektive auf makroökonomisches Wissen offeriert, die sich gleichermaßen von linearen Fortschrittsnarrativen wie von pauschalen Verfallsgeschichten absetzt. Sie stellt eine Grundlage für weiterführende soziologische Untersuchungen bereit und erlaubt auch eine bessere Einschätzung der aktuellen Debatten um eine Krise der Mainstream-Wirtschaftswissenschaften.

Schlüsselwörter: Soziologie ökonomischen Wissens und der Wirtschaftswissenschaften, Rationale Erwartungen, Neue Klassische Makroökonomik.

Abstract: The field of modern macroeconomics has not received much sociological attention. This neglect concerns for instance the work of Robert Lucas, that while being less visible and less politically influential than Friedmans and Hay-

*Kontaktperson: Hanno Pahl, Projektmitarbeiter am Soziologischen Seminar der Universität Luzern und assoziiertes Mitglied am Kolleg ,Postwachstumsgesellschaften“ der Friedrich-Schiller-Universität Jena, E-Mail: hanno.pahl@unilu.ch 
eks contributions - had an enormous impact on further developments in macroeconomics (Rational Expectations Revolution, New Classical Macroeconomics). In a more indirect manner, by proposing new modelling techniques and new standards of science, Lucas and his followers cultivated market-affirmative positions, including the proposition of a general policy-ineffectiveness. The text offers a pointed outline of core-developments in modern macroeconomics after the hegemony of the Keynesianism of the neoclassical synthesis, specifically addressing modelling techniques and changing visions of control. The perspective outlined is one that differs from linear narratives of progress as well as from simple stories of decay. It offers a possible starting point for more detailed sociological investigations into macroeconomic knowledge and enables a sounder appraisal of the recent debates about a crisis of mainstream economics.

Keywords: Sociology of Economic Knowledge and Economics, Rational Expectations, New Classical Macroeconomics.

If you cannot think outside the box, how do you know you're in the right box?

Axel Leijonhufvud

\section{Einleitung: Makroökonomik der Gegenwart als Anathema soziologischer Forschung}

Im Verlauf der ökonomischen Krise $2007 \mathrm{ff}$. gab es eine breitgefächerte Kritik bezüglich eines Versagens bzw. einer etwaigen Mitschuld der Mainstream-Wirtschaftswissenschaften angesichts der ungeahnten Krisenverwerfungen (siehe zur Debatte Caspari, Schefold 2011). Nach wenigen Jahren lässt sich feststellen, dass - bis dato - großflächige Strukturveränderungen innerhalb des Fachs ausgeblieben sind. Zwar kann von einer größeren Marktskepsis auch innerhalb des Mainstreams ausgegangen werden und mitunter haben neue Themenbereiche eine Konjunktur erlebt (zuletzt etwa die Frage der Vermögensungleichheit, siehe Piketty 2014). Auf der Ebene der wirtschaftspolitischen Steuerung wird das Paradigma des Inflation Targeting, welches zentralbankliches Handeln im Wesentlichen auf Geldwertstabilität enggeführt hat (Bernanke, Mishkin 1997), durch das breiter angelegte Konzept der sogenannten Macroprudential Regulation ergänzt, durch das auch systemische Risiken in der Finanzsphäre in den Verantwortungsbereich von Zentralbanken rücken sollen (Bank of England 2009). Alternative Forschungsrichtungen beziehungsweise Modellierungstechniken wie Agent $\mathrm{Ba}$ sed Modeling beginnen zunehmend auch in den Wirtschaftswissenschaften Fuß 
zu fassen (Farmer, Foley 2009). Aber der etablierte Theorien- und Methodenkanon des Mainstreams stand und steht innerhalb der Zentren des Fachs, insbesondere innerhalb der Makroökonomik, nicht ernsthaft zur Disposition. So wird an jener Modellklasse, die im Zuge der Krise von Kritikerseite als eine Art Hauptschuldiger identifiziert wurde (siehe exemplarisch Caballero 2010) - den DSGEModellen (Dynamic Stochastic General Equilibrium) - dezidiert festgehalten: Die Modifikation und Erweiterung von DSGE-Modellen um Aspekte wie Financial Frictions bildet seit der Krise eine der größten Wachstumsbranchen innerhalb der Mainstream-Makroökonomik (mehr dazu im Fortgang) und von diesen aktualisierten Modellen wird erneut nichts weniger erwartet als „to provide a workhorse setup for macroprudential analysis“ (Brzoza-Brzezina et al. 2011, S. 7).

Dieser Sachverhalt ist nicht erklärbar, ohne auf einige Geschehnisse in der jüngeren Vergangenheit des Fachs zu referieren, vor allem darauf, dass sich die Makroökonomik unmittelbar vor Ausbruch der Krise auf einem Zenit sowohl innerer wie nach Außen gerichteter Geschlossenheit wähnte, wie er seit den ersten Nachkriegsjahrzehnten und dem damaligen Regime des Keynesianismus der neoklassischen Synthese nicht mehr erreicht wurde. Mit Blick auf das theoretische Feld wurde eine neue neoklassische Synthese (Woodford 2009) ausgerufen, ein Konsens der Forschungsstränge von New Classical Macroeconomics und New Keynesian Economics, die sich in den 1980ern und 1990ern noch dezidiert konkurrierend gegenüberstanden. Auch für das Feld der geldpolitischen Beratung war von einem New Consensus (Goodfriend 2007) die Rede. Diese Entwicklungen wiederum lassen sich nicht aufschlüsseln, ohne der Bedeutung und dem Einfluss von Robert Lucas (Stichworte: Lucas-Critique, Rational Expectations Revolution, New Classical Macroeconomics) nachzuspüren, was im vorliegenden Text geschehen soll.

Es ist nicht unüblich, wenn Fachvertreter die Geschichte der Makroökonomik aktuell in zwei Abschnitte einteilen, „,vor Lucas (1976) und nach Lucas (1976)“ (Michaelis 2013, S. 1), oder dass von Lucas als „Architect of Modern Macroeconomics“ (Chari 1999, S. 2) gesprochen wird. Diese fachinterne Prominenz kontrastiert stark mit der weitgehenden Vernachlässigung der New Classical Macroeconomics und nachfolgender Entwicklungen seitens der Soziologie (und verwandter Beobachtungsposten): Soziologische Beiträge zur Kritik der Gegenwartsökonomik haben sich vor allem auf den Einfluss der Theorien von Friedman und Hayek als politisch einflussreichsten Stichwortgebern neoliberaler Umstrukturierungsprogramme bezogen. Dies ist nicht falsch, verkennt aber die (relativ) untergeordnete Position dieser beiden Figuren innerhalb der Disziplin der Wirtschaftswissenschaften. Hayek war innerhalb der Mainstream-Makroökonomik der zweiten Hälfte des 20. Jahrhunderts insofern ein Außenseiter, als sein Festhalten am formalisierungsskeptischen Programm der Austrian Economics gegenüber den Hauptlinien sämtli- 
cher Mainstream-Makroökonomik dieser Zeit (Gleichgewichtstheorie und Ökonometrie) stark konträr gelagert war (vgl. Backhouse 2000). ${ }^{1}$ Friedman war in erster Linie ein empirischer Ökonom, der in der pragmatischen Tradition Marshalls sowie des älteren US-amerikanischen Institutionalismus stand und selbst keine gewichtigen theoretischen Beiträge zur Fortschreibung der allgemeinen Gleichgewichtstheorie vorgelegt hat (vgl. Laidler 1999). ${ }^{2}$ Letztere Theorietradition bildet allerdings, in verschiedenen Ausprägungen, nach wie vor den theoretischen Kern der Mainstream-Makroökonomik, und es ist dieses Feld, das durch die Arbeiten von Lucas neue Impulse erhalten hat (man könnte hier auch von einer Radikalisierung sprechen). Die durch Lucas ausgelösten methodischen Entwicklungen, die den Mainstream im Bereich der Makroökonomie heute wesentlich bestimmen, lassen sich durchaus als „Schlag ins Kontor der herkömmlichen Makroökonomik, egal ob keynesianisch oder monetaristisch geprägt“ (Michaelis 2013, S. 3), interpretieren.

Der vorliegende Beitrag soll etwas Licht in das Dunkel moderner Makroökonomik bringen. Er ist insofern als explorativer Text zu verstehen, als ein sehr großer Bogen geschlagen wird, was notwendigerweise nicht nur an vielen Stellen auf Kosten von Details geht, sondern auch eine recht starke Selektivität erfordert. Angesichts der zu verzeichnenden weitgehenden Aussparung der New Classical Macroeconomics (und anschließender Entwicklungen) aus dem Sichtfeld soziologischer Beobachtung scheint ein solches Vorgehen legitim. Die offerierte „Gesamtschau“ soll nicht den Eindruck der Vollständigkeit erwecken, sondern als Anregung und Orientierungsmarke für weitere, spezifischer ansetzende Analysen dienen. ${ }^{3}$ Schwerpunkte werden zum einen auf (den Wandel von) Steuerungsvor-

1 Symptomatisch ist in dieser Hinsicht das folgende Urteil von Lucas (in Klamer 1984, S. 56): „The ideas of Hayek and Mitchell are interesting - worth building on - but their methods are not“. Im gleichen Interview heisst es auch: „The only way I feel I understand something is if I can write it down in a model and make it work" (ebenda, S. 49).

2 Der vorherrschende soziologische Fokus auf Friedman scheint insofern gerechtfertigt oder verzeihlich, als die politischen Implikationen der durch Lucas induzierten New Classical Macroeconomics vielfach ähnlich zu jenen des Friedmanschen Monetarismus ausgefallen sind (siehe Hoover 1984 und den Fortgang). Die folgenreichen methodischen Veränderungen lassen sich so allerdings nicht erhellen. Lucas (1977, S. 25) flaggt sein Ziel beispielsweise aus als ,attempt to understand and make more explicit the implicit model underlying the policy proposals of Henry Simons, Milton Friedman, and other critics of activist aggregative policy“.

3 In den letzten Jahren ist ein deutlicher Anstieg von Arbeiten auf dem Feld einer Sociology of Economics zu verzeichnen. Sei es dass die Entwicklung ökonomischen Denkens über lange Zeiträume ländervergleichend eruiert wurde (Fourcade 2009) oder qua Feldtheorie die ökonomische Forschungslandschaft eines einzelnen Landes detaillierter abgebildet wurde (Lebaron 2000 für Frankreich). Insbesondere im Bereich der Social Studies of Finance wurden auch kognitive Aspekte ökonomischer Wissensformationen mikrologisch thematisiert (MacKenzie 2008). Eine gute Übersicht findet sich bei Maeße (2013, S. 9 ff.). 
stellungen gelegt, weil sich die Makroökonomik als wichtiges Segment der Wirtschaftswissenschaften durch eine große Nähe zur Politik auszeichnet, zum anderen werden Fragen der Modellierbarkeit zentral gestellt, weil diese Forschungsweise das gesamte Feld der Mainstream-Wirtschaftswissenschaften seit der Mitte des 20. Jahrhunderts eindeutig dominiert.

Zum Argumentationsgang: Es wird eingesetzt (Teil 2) mit einer Kurzcharakterisierung des sogenannten Keynesianismus der neoklassischen Synthese (2.1.), der - als hegemoniale makroökonomische Wissensformation der ersten Nachkriegsjahrzehnte - für Lucas und seine Mitstreiter ein zentrales Abgrenzungsobjekt dargestellt hat (2.2.). Daraufhin (Teil 3) werden zentrale Aspekte der New Classical Macroeconomics konturiert. Entlang von drei Themenkomplexen wird nachvollzogen, inwieweit die dortigen Auffassungen als Zurückweisungen Keynesscher Annahmen und als Rückkehr zu oder gar Radikalisierung von klassischneoklassischen Prinzipien des Laissez-Faire auf neuartiger modelltheoretischer Grundlage gelten können. Die New Classical Macroeconomics stellen zwar einen signifikanten Einschnitt in der Entwicklung der makroökonomischen Forschung dar, aber selbstverständlich keinen Endpunkt. In Teil 4 wird aus diesem Grund zunächst (4.1.) das Forschungsprogramm der New Keynesian Economics kurz beleuchtet, das sowohl durch methodische (modellarchitektonische) Kontinuitäten als auch sachlichen Dissens zu den New Classical Macroeconomics geprägt ist. Ein ähnlicher Stellenwert kommt Teil 4.2. zu, in dem die Frage verhandelt wird, in welcher Weise der gegenwärtige makroökonomische Mainstream, verstanden als Schulterschluss oder Kompromiss beider Forschungslinien (neue neoklassische Synthese), auf die Krisenerscheinungen $2007 \mathrm{ff}$. reagiert. Im Resümee (Teil 5) werden zunächst die durch Lucas induzierten nachhaltigen Effekte innerhalb und außerhalb der Disziplin erörtert. Sodann geht es um die allgemeinere Frage, durch welche Muster sich die Entwicklung der modernen Makroökonomik auszeichnet. Hier wird besonders auf die modellinduzierte Etablierung kognitiver Pfadabhängigkeiten abgestellt, die nicht nur den Fortgang des Wissens, sondern auch die Brechungsstärke der Ökonomik gegenüber Impulsen von Außen maßgeblich bestimmen.

\section{Der hydraulische Keynesianismus der neoklassischen Synthese als Hintergrundfolie und Absetzungsobjekt}

Die Rational Expectations Revolution und der Aufstieg der New Classical Macroeconomics können nicht ohne Kontextualisierung erschlossen werden, und dies 
impliziert wenigstens einen kursorischen Blick auf die vormals dominante Formation makroökonomischen Wissens, den Keynesianismus der neoklassischen Synthese und dessen Entstehungszusammenhang.

\subsection{Die Gesamtwirtschaft als Interventionsobjekt wissenschaftlich angeleiteter Wirtschaftspolitik: Basale Charakteristika der Keynesian Economics}

Die General Theory of Employment, Interest and Money (Keynes 1936) gilt als Geburtsstunde einer dezidierten Makroökonomik und wurde auf breiter Basis als Antwort willkommen geheißen ,to fill the yawning gap between economic analysis and the real-world problem of the Great Depression. It offered a plausible explanation and a feasible course of action“ (Hoover 1988, S. 9). Sachlich bezog sich die gewichtigste Intervention von Keynes auf die Möglichkeit mangelnder effektiver Nachfrage (formal ausgedrückt: der Existenz eines Unterbeschäftigungsgleichgewichts), womit dem sogenannten Sayschen Gesetz (,jedes Angebot schafft sich seine Nachfrage“) widersprochen wurde, das zum Kerntheoriebestand sowohl in der klassischen als auch in der neoklassischen Ökonomik zählte. Mit Bezug auf wirtschaftspolitische Maßnahmen erweiterte Keynes den „range of possible stabilization policies to include fiscal as well as monetary measures“ (Mehrling 1998, S. 299), weniger im Sinne einer gänzlichen Neuerfindung (entsprechende Maßnahmen wurden bereits zuvor praktiziert, beispielsweise im Rahmen des New Deal), sondern als wissenschaftliche Unterfütterung und Legitimierung staatlicher Interventionspraktiken.

Allerdings war die ursprüngliche Form dieser Theorie, die ein Konglomerat aus anspruchsvollen Argumentationslinien und Formeln darstellte, nicht ohne Weiteres mit den Erwartungshaltungen des sich in den 1930er Jahren herausbildenden makroökonomischen Mainstreams kompatibel, so dass es bereits kurz nach der Veröffentlichung der General Theory zu verschiedenen Versuchen der „Übersetzung“ und „Vereindeutigung“ des komplexen Buchs kam. Keynes selbst hatte sich aus guten Gründen geweigert, seiner Gesamttheorie im Rahmen damals verfügbarer Formalisierungsmodi eine streng mathematische Gestalt zu geben, ${ }^{4}$ Vorbehalte, die seitens vieler seiner Nachfolger nicht mehr gehegt wurden. For-

\footnotetext{
4 Dazu vermerkt Backhouse (1997, S. 34): „To construct a formal model was to attempt to specify exactly what was and what was not to be included in the analysis - to be 'perfectly precise'. But if the world was vague and complex, such an approach was inappropriate. Thus, though Keynes used mathematics in The General Theory, he refused to use a mathematical model to summarize the argument as a whole“.
} 
malisierungsversuche wie das IS-LM-Modell, die in weitaus größerem Ausmaß zur Referenzfolie Keynesianischer Makroökonomik avancierten als die originären Keynesschen Überlegungen, haben sich dadurch ausgezeichnet, gemutmaßte Kerngehalte der General Theory im Rahmen eines gleichgewichtstheoretischen Forschungsrahmens ${ }^{5}$ darzustellen, weil die Gleichgewichtstheorie in den 1930er Jahren weithin als sicheres Fundament von Ökonomik als Wissenschaft aufgefasst wurde. Das IS-LM-Modell (Investment-Saving/Liquidity preference-Money supply) modelliert die Volkswirtschaft in Form eines gesamtwirtschaftlichen Gleichgewichts (resultierend aus einer Kombination von Gleichgewichtsmodellen für den Gütermarkt und für den Geldmarkt). Es basiert auf einer Reduktion der General Theory in ein einfaches System aus drei Gleichungen, die ihr Konstrukteur, John Hicks, mit einem ähnlichen Gleichungssystem vergleicht, das ihm zufolge die neoklassische Position abbildet. So gelingt es Hicks (und den Keynesianischen Makroökonomen in seinem Gefolge) zwar, einige Besonderheiten der Keynesschen Theorie rigoros abzubilden. Dies gelingt allerdings um den Preis, die General Theory auf ein deterministisches System von Simultangleichungen zu reduzieren - auf einen Spezialfall innerhalb des Kosmos der Walrasianischen Gleichgewichtstheorie - und alle Theoriebestandteile $\mathrm{zu}$ exkludieren, die sich dieser Formalisierungstechnik sperren. Fourcade (2009, S. 160) resümiert die Geschehnisse in folgender Weise: „Keynes’s economics was exported from Britain to the United States in the 1930s, it was then marketed back to Europe as ,Keynesian economics“ in the 1940s and 1950s“.

Die hieraus resultierende und in den ersten Nachkriegsjahrzehnten hegemoniale Form von Makroökonomik lässt sich mit Coddington (1976) als hydraulischer Keynesianismus bezeichnen, oder auch als Keynesianismus der neoklassischen Synthese (Togati 1998). Das Attribut „hydraulisch“ referiert auf die Annahme stabiler funktionaler Relationen zwischen makroökonomischen Aggre-

5 Die schrittweise Transformation der Gleichgewichtstheorie von einem spezifischen Forschungsprogramm zu einem disziplinbeherrschenden Paradigma muss hier vollständig ohne Erläuterung bleiben. Besonders lesenswert sind Mirowskis (1999) Überlegungen bezüglich massiver Konzeptübernahmen aus der Physik, deren Kernthesen bei Mitchell (1998, S. 85f.) wie folgt zusammengefasst werden: "[S]cientific economics was created in the 1870 s by translating the new language and imagery of physics into a vocabulary and set of metaphors for imagining the field of economic processes. The terminology of the new discipline - words like equilibrium, stability, elasticity, inflation, expansion, contraction, distribution, movement, friction - was borrowed intact from physics. And the central concept of economics, individual utility, was modelled directly on the new idea of energy. It represented the same unique, protean, unknowable force, giving the elements of the economic field their animation. Economics also borrowed from physics its models of explanation, in particular the prescription to express causal relations in mathematical form". 
gatgrößen (wie der gesellschaftlichen Gesamtnachfrage und der Arbeitslosenrate). Den damit verbundenen Steuerungsoptimismus verdeutlicht beispielsweise ein Zitat von Lawrence Klein (1966, S. 180), der sich in den 1950er und 1960er Jahren um die ökonometrische Fundierung der Keynesian Economics verdient gemacht hat: „There is no reason why intelligent economic planning cannot be of just the correct amount, that amount which gives permanent full employment and stable prices". Wirtschaft wird als zwar eigenlogisch operierende Sphäre dezentraler Entscheidungseinheiten gedacht, eine staatliche Kontextsteuerung ist aber tendenziell dazu in der Lage, Zustand und Verhalten des Gesamtsystems durch eine Manipulation von Aggregatgrößen zielgerichtet zu beeinflussen. Die Bezeichnung „neoklassische Synthese“ verweist auf verschiedene Dimensionen einer Kompromissbildung zwischen klassisch-neoklassischen und keynesianischen Argumentarien, der Nachfragesektor wurde vermittelst des IS-LM-Modells modelliert, der Angebotssektor (Arbeitsmarkt und Produktionsfunktion) hingegen entlang der Vorgaben des klassisch-neoklassischen ${ }^{6}$ Theoriebestandes. Dem IS-LM-Modell als „organizing theoretical apparatus of the emerging discipline of macroeconomics“ (De Vroey, Hoover 2004, S. 3) ist in dieser Konstellation auch insofern eine Zentralstellung zugekommen, als es den „ground on which the postwar intellectual battles were fought“ (Mehrling 2006, S. 71) bereitgestellt hat: In ihm konnten die einander in Teilen wiedersprechenden politischen Prämissen von neoklassischer Tradition und Keynesscher Theorie jeweils als Spezialfälle abgebildet werden, und selbst in der anschließenden Debatte zwischen Keynesianern und Monetaristen wurde das IS-LM-Modell vielfach als gemeinsames Spielfeld akzeptiert.

Ein letzter Punkt, der genannt werden soll, betrifft die Inkorporierung der sogenannten Phillips-Kurve in das Arsenal des hydraulischen Keynesianismus, in der - vereinfacht gefasst - ein Zusammenhang von Inflation und Arbeitslosigkeit behauptet wird, der ein Trade Off zwischen beiden Größen ermögliche. Zunächst empirisch als Korrelation (inverses Verhältnis zwischen Nominallohn und Unterbeschäftigung) für einen bestimmten Zeitraum festgestellt (Phillips 1958), avan-

6 Keynes selbst verwendet das Attribut „klassisch“ sowohl zur Kennzeichnung solcher Strömungen, die heute gemeinhin als klassische politische Ökonomie bezeichnet werden, als auch für Theorien, die man heute mit der Bezeichnung „neoklassisch“ versehen würde. Diese Ineinssetzung ist gerade unter methodischen Aspekten problematisch (weil die Arbeiten der klassischen politischen Ökonomie prominent auf arbeitswerttheoretische Argumente abstellen, wohingegen in der Neoklassik marginalistische Wertauffassungen obligatorisch sind). Legt man den Schwerpunkt allerdings auf politische Implikationen (und darum ging es Keynes an dieser Stelle) ist eine solche Unschärfe verzeihlich, weil sich in beiden Wissensformationen deutliche Präferenzen für eine Selbstregulierung von Märkten und gegen staatliche Eingriffe auffinden lassen. 
cierte die Phillips-Kurve zu einem zentralen Konzept wirtschaftlicher Steuerung. In der fünften Auflage (aus 1961) von Samuelsons Bestseller-Lehrbuch Economics heißt es (zitiert nach Pearce, Hoover 1995, S. 205):

The indicated ,Phillips Curve“ shows by its downward slope that increasing the level of unemployment can moderate or wipe out the upward price creep. There is, so to speak, a choice for society between reasonable high employment with maximal growth and a price creep, or reasonable stable prices with considerable unemployment; and it is a difficult social dilemma to decide what compromises to make. ${ }^{7}$

\subsection{Der Frontalangriff der Rational-Expectations-Schule}

Bis gegen Ende der 1960er Jahre prozessierte normalwissenschaftliche makroökonomische Forschung zu großen Teilen innerhalb der Koordinatensysteme der neoklassischen Synthese. Dass sich dies einer vollständig kohärenten Wissensformation verdankt hat, kann mit guten Gründen angezweifelt werden, so findet sich (als ein Beispiel von vielen) bei Greenwald und Stiglitz (1987, S. 3) folgender Hinweis auf die mangelhafte innere Konsistenz der neoklassischen Synthese:

The schizophrenia to which Keynesian economics gave rise was reflected in the way that economics was taught: micro-economic courses, in which students were introduced to Adam Smith's invisible hand and the fundamental theorems of welfare economics, were followed by macro-economic courses, focusing on the failures of the market economy and the role of the government in correcting them. Two sub-disciplines developed, with microeconomists looking down upon the (lack of) rigor of the macro-economists, and denigrating the lack of theoretical foundations, while macro-economists castigated micro-economists for the obvious inappropriateness of their theories.

Trotz dieser Bruchlinien, von denen die genannte Inkompatibilität zwischen Mikroökonomik und Makroökonomik nur ein Beispiel darstellt, kam es zunächst nicht zu einer wissenschaftlichen Revolution (Kuhn 1962). Durch die monetaristischen Strömungen wurde zwar die vormalige Fokussierung auf Nachfragesteuerung herausgefordert und es wurden angebotsseitige Überlegungen auf die Agenda gesetzt. Aber wie oben vermerkt fanden die entsprechenden Debatten oftmals innerhalb des Varianzraums der neoklassischen Synthese statt. ${ }^{8}$ Auch empirische

7 Bis heute ist der Ausspruch des ehemaligen Bundeskanzlers Helmut Schmidt bekannt: „Mir scheint, daß das Deutsche Volk - zugespitzt - 5\% Preisanstieg eher vertragen kann, als 5\% Arbeitslosigkeit“ (in: Süddeutsche Zeitung, 28. Juli 1972, S. 8).

8 Theoriezentrierte Arbeiten wie Leijonhufvuds (1968) Buch On Keynesian Economics and the Economics of Keynes, in denen die gegenüber Keynes abweichenden Erklärungsmodi der Keynesi- 
Ereignisse - der sogenannte Ölpreisschock 1973 (als Schock auf der Angebotsseite) und die sich manifestierende Unfähigkeit der Wirtschaftspolitik, in diesem Umfeld zielgerichtet zu agieren (Stagflation) - können nicht als alleinige Faktoren veranschlagt werden, auch wenn sie zur Erosion der Hegemonie der neoklassischen Synthese beigetragen haben.

Lucas und seine Mitstreiter konnten das Profil ihrer eigenen Forschungsperspektive rationaler Erwartungen nicht zuletzt dadurch schärfen und mit Relevanz ausstatten, dass sie die Konzepte und Ordnungsvisionen des hydraulischen Keynesianismus der neoklassischen Synthese auf ganzer Bandbreite attackiert haben. Dies betrifft sowohl die epistemischen Zusammenhänge der dortigen Theorie- und Modellarchitekturen als auch die hieraus abzuleitenden wirtschaftspolitischen Empfehlungen. Am deutlichsten manifestiert sich dies rhetorisch im Text After Keynesian Macroeconomics (Lucas, Sargent 1979), der im Titel offensiv die Marschrichtung verrät. ${ }^{9}$ Der Beitrag ist zu einem Zeitpunkt entstanden, als bereits erste wichtige Grundlagenarbeiten der Rational Expectations-Schule erschienen waren ${ }^{10}$ und die wirtschaftliche Entwicklung zudem durch das Phänomen der Stagflation und den zweiten Ölpreisschock systematische Grenzen Keynesianischer Konjunktursteuerung offenbart hatte. Entsprechend selbstbewusst verkünden Lucas und Sargent (1979, S. 1), dass die herrschende Doktrin ihrer Auffassung nach „fundamentally flawed“ sei. Als Programm markieren sie „to sort through the wreckage, determining which features of that remarkable intellectual event called the Keynesian Revolution can be salvaged and put to good use and which others must be discarded“, wobei es auch um ein „reopening of basic issues in monetary economics which have been viewed since the thirties as ,closed““ gehen müsse. Bereits vorwegnehmend halten sie fest (ebenda, S. 2): „our intent is to establish that the difficulties are fatal: that modern macroeconomic models are of no value in guiding policy“. Der tradierte Bestand makroökonomischen Wissens wird als Trümmerhaufen charakterisiert, als eine unwiederbringlich erschütterte Ordnungskonstellation.

an Economics präzise problematisiert wurden, fanden eine gewisse Aufmerksamkeit, die daran anschließenden Alternativprogramme (zuvorderst die sogenannten Disequilibrium Macroeconomics) konnten sich aber nicht dauerhaft behaupten.

9 In einem Interview hat Lucas später auf die Bemerkung, wonach dieser Beitrag „, lot of powerful rhetorical statements“ (Snowdon, Vane 2005, S. 282) enthalte, und ob diese bewusst verwendet wurden, geantwortet: „Yes. We were invited to a conference sponsored by the Boston Fed. In a way it was like being in the enemy camp and we were trying to make a statement that we weren't going to be assimilated“.

10 Dies waren vor allem die Texte Expectations and the Neutrality of Money (Lucas 1972) und Econometric Policy Evaluation: A Critique (Lucas 1976). 
Sämtliche Modelle Keynesianischer Provenienz könnten nicht berücksichtigen, dass sich die Erwartungsstrukturen ökonomischer Akteure an wirtschaftspolitische Maßnahmen anpassen respektive diese antizipieren. Berücksichtigt man dies, sei es nicht länger möglich, invariante funktionale Relationen zwischen makroökonomischen Aggregatgrößen zu unterstellen, was eine basale Grundlage der Keynesianischen Steuerungsvision war (vgl. Backhouse 2010, S. 131ff.). Geht man, wie Lucas (1972, 1976) im Anschluss an Muth (1961) argumentiert, von konsequent rational-erwartenden Akteuren aus, deren interne Modelle sich im Einklang mit der Realität befinden (Rational Expectations Hypothesis), laufen wirtschaftspolitische Maßnahmen nahezu zwangsläufig ins Leere. Wird zum Beispiel eine Erhöhung der Geldmenge erwartet, gehen die Akteure in ihren Kalkulationen von einem Anstieg des allgemeinen Preisniveaus aus, so dass die zur Stimulierung wirtschaftlicher Aktivitäten gedachte Maßnahme lediglich einen Einfluss auf das nominale Preisniveau hat (dies führt zur sogenannten PolicyIneffectiveness Proposition). ${ }^{11}$

Es kommt zu einer Verschränkung von Theorieebene und Gegenstandsebene. Nicht nur Ökonomen werden als Modellbildner gedacht, das Gleiche wird nun auch für die Wirtschaftsakteure angenommen. Es wird unterstellt, dass es ein richtiges Modell der Wirtschaft gibt und dass das darin inkorporierte Wissen allen Teilnehmern am Wirtschaftsprozess zur Verfügung steht:

That is, in a rational-expectations equilibrium, everyone's expectations about what will happen are consistent with the macroeconomic forces actually at work, and also consistent with everyone else's expectations, no matter what kinds of policies are pursued (Howitt 2006, S. 349).

Gemeint ist nicht, dass die Wirtschaftsakteure ihr Handeln an einem wahren Modell der Ökonomie orientieren, das ihnen faktisch als Kalkulationsrahmen zur Verfügung steht, sondern dass ,they adjust their behaviour to obvious errors until they act as if they knew the true model“ (Hoover 1988, S. 143). ${ }^{12}$ Das Argument ist mitunter ein statistisches:

11 Oder mit etwas anderem Fokus bei Mehrling (2011, S. 88) ausgedrückt: „It is simply a logical mistake, argued Lucas, to treat the behavioral equations in an economic model as invariant to policy intervention, since agents should optimally use whatever they know about current policy practice when they are deciding how to behave. Change the policy rule and you change the behavior rule as well".

12 Ähnlich hatte sich bereits Schumpeter (2009, S. 1225) zur Walrasianischen Gleichgewichtstheorie geäußert: Es wird angenommen, „dass die Wirtschaftssubjekte auf dem Markt zwar keine Gleichungen lösen, aber dennoch mit anderen Mitteln das Gleiche tun, was der Theoretiker durch die Lösung von Gleichungen vollzieht““. 
The rational expectations model assumes that economic actors make decisions based on all available information and are therefore able, at least on average, to accurately predict the future from a present point in time. Errors in actor estimations are randomly distributed and are thus insignificant for average outcomes (Beckert 2014, S. 8).

Es bleibt gleichwohl fraglich, ob dies eine kohärente Modellierungsstrategie darstellt. Unter der Hand wird mit dem Theorem rationaler Erwartungen - ähnlich wie bereits beim Walrasianischen Auktionator der Fall - ein Synthesekonzept eingeschleust, das den individualistischen Prämissen des Theorieprogramms widerspricht. Sargent (zitiert nach Schlefer (2012: XV)), ein Verfechter der Theorie rationaler Erwartungen, gesteht dies mit einer Formulierung wie „communism of beliefs“ auch mehr oder minder ein. Der Siegeszug der damit eingeleiteten und im Folgenden näher zu betrachtenden New Classical Macroeconomics verlief - jedenfalls im akademischen Kontext - äußerst rasant und breitflächig. Für die Zeit Anfang der 1980er Jahre vermerkte Blinder (1997, S. 109f.): „By about 1980, it was hard to find an American academic macroeconomist under the age of 40 who professed to be a Keynesian. That was an astonishing intellectual turnabout in less than a decade - an intellectual revolution for sure“.

\section{Steuerungsabstinenz als Ordnungsvision: Konturen der New Classical Macroeconomics}

Lucas und seinen Kollegen ist es gelungen, durch das konsequente GeltendMachen eines einzigen zentralen Faktors, der Erwartungsdimension, das tradierte makroökonomische Wissen mehr oder minder handstreichartig zu entwerten und das Feld für eigene Ordnungsfiguren freizumachen. Was als Rational Expectations Revolution begann hat sich zügig zu einer neuen makroökonomischen Wissensformation entfaltet, den New Classical Macroeconomics, in denen klassisch-neoklassische Argumentarien auf neuer modelltheoretischer Grundlage aktualisiert wurden. Im folgenden Abschnitt sollen entlang von drei Dimensionen eine Reihe der neuen Theoriefiguren und Zusammenhangsbehauptungen vorgestellt werden. Diese Abhandlung ersetzt keine Detailstudien zu ökonomischen Sachfragen, sondern ist bemüht, die Hauptlinien im Wandel dominanter ökonomischer Wissensformationen möglichst plastisch zu machen, ohne die Komplexität über Gebühr ansteigen zu lassen. 


\subsection{Triebkräfte von Konjunkturzyklen und Ursachen von Arbeitslosigkeit}

Periodische Schwankungen zentraler wirtschaftlicher Gesamtgrößen („Konjunkturzyklen“) gehören zu den empirisch am besten dokumentierten Phänomenen der modernen kapitalistischen Wirtschaft. Das gleiche gilt für die (mindestens periodische) Existenz von Arbeitslosigkeit. Für die Walrasianische Tradition stellten sowohl Konjunkturzyklen als auch die Existenz von Arbeitslosigkeit ein Erklärungsproblem dar, weil beide Phänomene unter der Voraussetzung des dortigen Theorierahmens eigentlich nicht vorkommen sollten. Mit Keynes’ General Theory erhielt die gesamtwirtschaftliche Nachfrage eine Schlüsselstellung zur Erklärung der Expansion und Kontraktion von Produktion und Beschäftigung. Einzelne Phasen von Konjunkturzyklen gelten als Ungleichgewichtszustände, etwa wenn im Arbeitsmarkt das Angebot an Arbeitskräften größer ist als die Nachfrage und es zu unfreiwilliger Arbeitslosigkeit kommt. An sich nutzbare Ressourcen liegen aufgrund von Nachfrageschwankungen brach, der Staat sei gefordert, um das System durch zusätzliche Nachfrage zu stimulieren und auf einem höheren Aktivitätsniveau zu stabilisieren (vgl. Hoover 1992, S. 84; Iversen, Soskice 2006, S. 427 f.). Während bei Keynes selbst die Frage von Erwartungsbildungen unter Unsicherheit zentral gestellt wurde (unter anderem vermittelst des nicht-formalisierten Konzepts der sogenannten Animal Spirits), kam es im Zuge der neoklassischen Synthese zu einer Verschiebung im Erklärungsmodus:

Because it had abandoned uncertainty as an explanation for the volatility of investment and output, the Keynesian models of the post-war period depended on the ad hoc assumption of price rigidities in order to be able to explain the persistence of unemployment in an otherwise Walrasian economy (Braun 2014, S. 59). ${ }^{13}$

Findet sich bei Keynes ein nicht-formalisiertes Erwartungskonzept mit starkem Zukunftsbezug, so rekurrierten die Protagonisten des Keynesianismus der neoklassischen Synthese auf ein im Rahmen ihres Walrasianischen Forschungspro-

13 Was als bloße modelltheoretisch-induzierte Rationalisierung intendiert war, führte mitunter zu politisch brisanten Verschiebungen: „An die Stelle der Keynesschen Vorstellung einer Hierarchie von Märkten tritt die in der Neoklassik übliche simultane Gleichgewichtslösung, wobei starre Geldlöhne dafür verantwortlich sind, daß der Reallohn nicht auf ein Niveau sinkt, das den Zustand der Unterbeschäftigung beseitigt. Flexible Geldlöhne und flexible Preise, so suggeriert die Neoklassische Synthese, führen mithin automatisch zu Vollbeschäftigung, wenn sich das System nicht in der Liquiditäts- oder in der Investitionsfalle befindet. Derweil Hicks lediglich wichtige Aspekte der Allgemeinen Theorie vernachlässigt, stellt die Neoklassische Synthese die Überlegungen von Keynes damit auf den Kopf“ (Eicker-Wolf 2003, S. 23f.). 
gramms leichter $\mathrm{zu}$ formalisierendes Konzept adaptiver Erwartungen. ${ }^{14}$ Die dadurch zunächst ausgeschlossene Volatilität des ökonomischen Systems wurde über den Kunstgriff der Einfügung von Rigiditäten zurückgewonnen.

Im Gefolge der Rational Expectations Revolution gab es eine nochmals ganz andersgeartete Interpretation des Phänomens, dass ökonomische Zeitreihendaten regelmässige Schwankungen in ihren Kerngrößen aufweisen. Ökonomische Fluktuationen werden als optimale Reaktionen auf unstetig verlaufende Prozesse technologischen Wandels begriffen, ein Ansatz, der in den sogenannten Real Business Cycle-Theorien ab Anfang der 1980er Jahre zur Entwicklung einer neuartigen, in Zukunft äußerst wirkungsmächtigen Modellklasse führte (Kydland, Prescott 1982). Gleichgewichtszustände werden nicht länger mit Ungleichgewichtszuständen kontrastiert, sondern die Wirtschaft gilt als ein permanent sich im Gleichgewicht befindendes System:

The bulk of economic fluctuations observed [...] could be interpreted as an equilibrium outcome resulting from the economy's response to exogenous variations in real forces (most importantly, technology), in an environment characterized by perfect competition and frictionless markets (Galí 2008, S. 3).

Die sich in Zyklen manifestierende Dynamik der kapitalistischen Wirtschaft wird auf externe Faktoren verschoben, auf diese Weise wird die empirische Erscheinung mit den gleichgewichtstheoretischen Prämissen harmonisiert, ohne wie im Fall der neoklassischen Synthese - auf Marktfriktionen abstellen zu müssen.

Fluktuationen der Beschäftigungsrate (Arbeitslosigkeit) gelten nicht länger als möglicher Weise unfreiwilliger Struktureffekt von Ungleichgewichtszuständen, sondern als ,the product of the utility-maximizing choices of rational economic agents“ (Hoover 1988, S. 29). Entsprechend hat der Diskurs der New Classical Economics keine Verwendung mehr für eine Unterscheidung von freiwilliger und unfreiwilliger Arbeitslosigkeit. Wirtschaftspolitische Implikationen betreffen eine rigorose Ablehnung von Keynesianischem Nachfragemanagement (,costly efforts at stabilization are likely to be counterproductive“, heisst es bei Prescott 1986, S. 21), ${ }^{15}$ und auch die Geldpolitik wird darauf verpflichtet, möglichst wenig in das Marktsystem einzugreifen:

14 Adaptive Erwartungen bezeichnen Erwartungsstrukturen, die sich primär an beobachteten Werten (ökonomischer Variablen) aus der Vergangenheit orientieren. Bis zur Lucas-Kritik war dieser Typ der Erwartungsmodellierung in den Wirtschaftswissenschaften vorherrschend (siehe Evans, Ramey 2001).

15 In dieser Hinsicht gehen die Prämissen weit über den Friedmanschen Monetarismus hinaus, der das antizyklische Projekt als solches nicht in Frage gestellt, sondern lediglich eine (radikal) 
[T]he New Classicals' advice to policymakers is straightforward: do not try any surprise moves. Choose a simple money growth plan consistent with your inflation goals. Announce the plan far enough in advance to allow markets to react. Then just follow the plan (Stark, Taylor 1991, S. 21).

Fiskal- und Geldpolitik werden nicht mehr als Möglichkeiten adressiert, die Ergebnisse von Marktprozessen zu verbessern, sondern als Instanzen, die im besten Fall wirkungslos sind, oftmals aber auch zu einer wohlstandsschädlichen Verzerrung von Marktprozessen führen.

\subsection{Die Zeitdimension: Priorisierung der langen Frist bzw. Einbezug der Differenz von kurzer und langer Frist}

Innerhalb der Wirtschaftswissenschaften hat sich bereits mit den Arbeiten David Ricardos ein diachrones Ordnungsschema etabliert, das bezüglich der Entwicklung kapitalistischer Wirtschaften zwischen kurzer (short run) und langer Frist (long run) unterscheidet (vgl. Jonas 1964, S. 111). Keynes (1924, S. 65) formulierte in seiner Auseinandersetzung mit der Quantitätstheorie den später berühmt gewordenen Satz „In the long run we are all dead“. Er kritisierte darin die Annahme, wonach eine staatliche Manipulation der Geldmenge (etwa qua Deficit Spending) keine Effekte habe, weil sich das Wirtschaftssystem in der langen Frist an den neuen Zustand anpassen würde und es lediglich zu einer Änderung nominaler Größen kommen würde. Die neoklassische Synthese kann als Versuch einer Kompromissbildung in der Gewichtung beider Zeitdimensionen und ihrer Gesetzlichkeiten begriffen werden: ${ }^{16}$

The long run domain was given to the Classicals, since wages and prices were assumed flexible in the long run and the neoKeynesians had already agreed that with flexible wages and prices the economy would gravitate to its long run equilibrium. For policy-oriented Keynesian macro economists, these new terms of synthesis were still acceptable, since there was still a short run role for their primary policy concern: stabilization policy (Colander 2002, S. 5).

Bei Lucas (in Snowdon, Vane 2005, S. 277) werden die Bemühungen seitens der New Classical Macroeconomics als Radikalisierung einer Rückeroberung Keyne-

andere Auffassung bezüglich der geeigneten Hebel verfochten hat (Geld- versus Fiskalpolitik, Rules versus Discretion, siehe dazu Mehrling 2011, S. 145).

16 Ein gutes Beispiel hierfür bildet wiederum das IS-LM-Modell, das beide Fälle abbilden kann. 
sianischen Terrains durch die Gleichgewichtstheorie beschrieben: „Samuelson’s neoclassical synthesis reclaimed the long run for neoclassical analysis, at least here in the USA. Now Samuelson's students - my whole generation - are trying to get the short run back, too!“. Der vormalige Burgfrieden wurde aufgekündigt, oder, mit Hoover (1988, S. 30) formuliert, „rational expectations collapses the short run into the long run“. Für die Theoretiker der New Classical Macroeconomics macht das Differenzschema von kurzer und langer Frist bereits als solches keinen Sinn, weil sie immer schon unter der Prämisse der Markträumung operieren. In der Tendenz läuft eine solche Homogenisierung der Temporalstrukturen zudem auf eine Beseitigung der Differenz von Mikro- und Makroökonomie hinaus. Hoover (1988, S. 87) spricht mit drastischen Worten von einer „euthanasia of macroeconomics“, ein Aspekt, der bei Lucas (1987, S. 107f.) als dezidiertes Ziel ausgewiesen wird: „[T]he term ,macroeconomic‘ will simply disappear from use and the modifier ,micro' will become superfluous. We will simply speak [...] of economic theory“. Die im Zuge der Keynesschen Intervention zu Tage getretene Notwendigkeit eines eigenständigen makroökonomischen Kategorienapparats wird nun als bestenfalls temporär gerechtfertigte Anomalie bewertet, die im Zuge systematischer Wissensentwicklung wieder zurückgenommen werden muss.

\subsection{Konjunkturzyklen und langfristiges Wirtschaftswachstum: Relevanzverschiebungen}

Das vermerkte Kollabieren (respektive die Beseitigung) der Unterscheidung von kurzer und langer Frist, die Neuinterpretation des Phänomens der Konjunkturzyklen sowie die Ablehnung eines eigenständigen makroökonomischen Analyserahmens sind zugleich mit einer gewichtigen Akzentverschiebung in der Forschung verbunden. Die kurze Frist war, wie gezeigt, seit jeher dem Problem von Konjunkturzyklen und Arbeitslosigkeit gewidmet, wohingegen es in der langen Frist vornehmlich um die Frage von Wirtschaftswachstum geht. Es ist wenig verwunderlich, dass sich Lucas nach seiner Grundlagenkritik am Keynesianischen Demand Management im Verlauf der 1980er Jahre schwerpunktmässig der Wachstumstheorie zugewandt hat und neben Paul Romer zu einem wichtigen Vertreter der New Growth Theory avancierte (siehe etwa Lucas 1988). Deutlich wird diese Schwerpunktverlagerung zum Beispiel in einer Interviewpassage, in der Lucas die wirtschaftlichen Entwicklungen des 20. Jahrhunderts gewichtet: „I think that economic growth, and particularly the diffusion of economic growth to what we used to call the Third World, is the major macroeconomic event of the twentieth century. But the Great Depression is a good second“ (Lucas in Snowdon, Vane 2005, S. 274). Galt die Große Depression lange Zeit als wichtigstes 
empirisches Phänomen, das die Existenz der Makroökonomik inklusive ihrer Steuerungsvisionen rechtfertigte, bemüht sich Lucas um eine deutliche Dezentrierung der Bedeutung dieses Ereignisses.

Wie verfestigt die Annahme fortan weitgehend krisenloser Entwicklungsverläufe im Lager der New Classical Macroeconomics war, zeigt eine Aussage, die Lucas (2003, S. 1) - wenige Jahre vor Ausbruch der aktuellen Krise - an prominenter Stelle getätigt hat (im Rahmen einer Presidential Address bei der Jahrestagung der American Economic Association): „My Thesis in this lecture is that macroeconomics [...] has succeeded: Its central problem of depression prevention has been solved, for all practical purposes, and has in fact been solved for many decades“. Den Hintergrund dieser Aussage bildete neben den disziplinären Konjunkturen vermutlich auch die Phase der sogenannten Great Moderation (Bernanke 2004), ein Begriff, der zur Kennzeichnung des Zeitraums ab Mitte der 1980er Jahre verwendet wurde, der sich - bis zum Ausbruch der aktuellen Krise - durch eine geringe Volatilität ökonomischer Kerngrößen ausgezeichnet hat, was oftmals auf neuartiges, durch Mithilfe der Mainstream-Ökonomik entstandenes, Design von Institutionen (vor allem Zentralbankunabhängigkeit) zurückgeführt wurde. Dass es im gleichen Zeitraum zahlreiche empirische Entwicklungen gab, die das harmonische und kategorisch optimistische Bild der Great Moderation korrigiert hätten (etwa sich häufende Finanzkrisen oder zunehmende Vermögensungleichverteilungen) ist ein gutes Indiz für theoretisch-induzierte Wahrnehmungsverengungen.

\section{Makroökonomik nach der Rational Expectations Revolution - Einige Schlaglichter}

Die Durchsetzung der New Classical Macroeconomics stellt einen signifikanten Einschnitt in der Entwicklung makroökonomischen Denkens dar, bildet aber keinen Endpunkt. Nachfolgende Entwicklungen sollen im Rahmen des vorliegenden Textes so weit skizziert werden, dass ein vorläufiges Gesamtbild des Einflusses der Rational Expectations Revolution ermöglicht wird.

\subsection{Kontinuitäten und Diskontinuitäten: Die New Keynesian Economics}

Das Forschungsprogramm der New Keynesian Economics (für einen detaillierten Überblick siehe Gordon 1990), dessen erste Grundlagenarbeiten in die späten 
1970er Jahre fallen und das spätestens seit einem prominenten Sammelband gleichen Namens (Mankiw, Romer 1991) als makroökonomische Wissensformation klar erkennbar ist, zeichnet sich durch eine doppelte Stellung gegenüber den New Classical Macroeconomics aus: Ihnen wird grundsätzlich darin gefolgt, dass von makroökonomischen Modellen eine Mikrofundierung eingefordert und die Theorie rationaler Erwartungen als Ausgangspunkt genommen wird. Allerdings wird der zweite Grundpfeiler der New Classical Macroeconomics, die These der Markträumung in der kurzen Frist (durch die Annahme vollständiger Preisflexibilität bzw. vollständiger Konkurrenz) verabschiedet oder kontingent gesetzt.

Dies führt dazu, dass die basalen Modellarchitekturen der New Keynesian Economics einerseits eine große Ähnlichkeit zu jenen Modellen aufweisen, die erstmalig als Real Business Cycle-Modelle den Kernbestand der New Classical Macroeconomics bildeten und die nun durch Einfügung verschiedener Marktfriktionen ergänzt wurden:

The development of new-Keynesian economics in the past decade has primarily involved the search for rigorous and convincing models of wage and/or price stickiness based on maximizing behavior and rational expectations (Gordon 1990, S. 1137). ${ }^{17}$

Andererseits resultieren aus der Abänderung der Annahme der Markträumung (zuvor ausgeschlossene) Spielräume für wirtschaftspolitische Interventionen: „When there are rigidities in price and wage setting, or when wages are collectively bargained, rational expectations are perfectly compatible with macroeconomic policies affecting production and employment“ (Iversen, Soskice 2006, S. $426 \mathrm{f}$.). Die Standardmodelle der New Classical Macroeconomics inclusive der These der Nicht-Effizienz jeglicher Geld- und Wirtschaftspolitik werden gleichsam zu Spezialfällen deklariert: „In the limiting case of perfect price flexibility [...] the cyclical dynamics resemble those of a real business cycle model, with monetary policy affecting only nominal variables“(Clarida, Gali et al. 1999, S. 1664f.).

So wie die hieraus resultierenden Modelle deutlich eklektizistische Züge tragen, je nachdem welche Arten von Friktionen eingeführt werden, stellt das Segment auch wirtschaftspolitisch ein eher heterogenes Konglomerat dar: „[N]ew Keynesians do not hold a unified view with respect to the relative potency of fiscal and monetary policy. [...] New Keynesians are not advocates of ,fine-tuning ' and the excessive policy activism of old-style Keynesian models“ (Snowdon, Vane 1997, S. 8). Durch den Einbezug von Erwartungsstrukturen ist der Weg zum Steuerungs-

17 Das dahinter stehende Modell imperfekter Konkurrenz geht beispielsweise davon aus, dass Firmen nicht nur Preisnehmer sind (wie in der Walrasianischen Tradition), sondern auch Preissetzer (vgl. Dixon 1997). 
optimismus der 1960er Jahre versperrt, gleichwohl werden mannigfache Situationen thematisiert, in denen das Prozessieren des Marktes zu nicht-optimalen Resultaten führt und durch Kontextsteuerung potenziell verbessert werden könnte.

\subsection{Neue Neoklassische Synthese, DSGE-Modellstandard und die Krise $2007 \mathrm{ff}$.}

Diese Entwicklungen erinnern in manchen Aspekten an die originäre neoklassische Synthese der Nachkriegsjahrzehnte, ${ }^{18}$ und in der Tat wurde (wie eingangs vorweggenommen) fast zeitgleich mit dem Ausbruch der Krise $2007 \mathrm{ff}$. eine neue neoklassische Synthese ausgerufen. Die Vertreter der New Classical Macroeconomics haben sich im Zeitverlauf in unterschiedlicher Weise zu den New Keynesian Economics positioniert. Äußerst kritisch äußerte sich beispielsweise Robert Barro (1989, S. 12), der hinter den dortigen Bestrebungen nichts weiter vermutete als ein politisch motiviertes Reverse Engineering: „Instead of providing new theoretical results and hypotheses for empirical testing, the objective often seems to be to provide respectability for the basic viewpoint and policy prescriptions that characterize the old Keynesian models“. Positiver, wenn auch in indirekter Weise, hat sich in der jüngeren Zeit Robert Lucas (in Hoover, Young 2011, S. 21) geäußert, wenn er die Entkopplung der Theorie rationaler Erwartungen von wirtschaftspolitischen Implikationen als positiven Entwicklungstrend rubriziert:

People thought rational expectations were associated with conservative policies. [...] And now no one thinks rational expectations is going to support conservative policies across the board. I think we've gone past that idea. It doesn't mean you have to be in love with rational expectations, but it's getting divorced from the political thing - at least it should be.

In welcher Weise wird nun seitens der Mainstream-Makroökonomik, verstanden als eine Art pragmatischer Burgfrieden zwischen New Classical und New Keynesian Economics, ${ }^{19}$ auf die Krisenverwerfungen seit 2007 Bezug genommen? Thomas

18 Dies gilt auch für die oben bereits am Beispiel der neoklassischen Synthese diskutierten problematischen Veränderungen zentraler Erklärungsmodi (verglichen mit der originären Keynesschen Theorie): „In the DSGE models now favored by central banks, business cycle movements in output and prices originate from price and wage stickiness. In order to reduce this kind of volatility more flexibility in prices and wages are required. That is why many central banks call for more flexibility. In a more flexible world, central banks will not be called upon so often to stabilize output, and thereby set price stability at risk" (Grauwe 2010, S. 480).

$19 \mathrm{Zu}$ welchen Anteilen die neue neoklassische Synthese tatsächlich einen Kompromiss und keine bloße Einheitsrhetorik darstellt kann hier nicht abschließend eruiert werden. Leijonhufvud (2009, S. 3f.) interpretiert die Sachlage wie folgt: „The lack of an alternative general theoretical 
Sargent (2010, S. 28) verweist auf den Charakter der Rational Expectations-Annahme als „workhorse assumption for policy analysis“ (ebenda: 29) bei Ökonomen mit ganz verschiedenartigen politischen Ansichten (hier werden als Beleg „linke“ New Keynesians wie Stiglitz angeführt). Der makroökonomische Mainstream sei de facto wesentlich pluralistischer als von Kritikerseite suggeriert, hinzukomme, dass die etablierten Modellklassen nicht für Krisenzeiten entworfen seien, sondern „to describe aggregate economic fluctuations during normal times when markets can bring borrowers and lenders together in orderly ways“ (ebenda). Eine ähnliche Position kommt auch in einer Formulierung bei Chari (2010, S. 9f.) zum Ausdruck, der im Rahmen einer Anhörungskommission der US-amerikanischen Regierung zum Zustand der Wirtschaftswissenschaften ausführte:

The recent crisis has raised, correctly, the question of how best to improve modern macroeconomic theory. I have argued we need more of it. After all, when the AIDS crisis hit, we did not turn over medical research to acupuncturists. In the wake of the oil spill in the Gulf of Mexico, should we stop using mathematical models of oil pressure? Rather than pursuing elusive chimera dreamt up in remote corners of the profession, the best way of using the power in the modeling style of modern macroeconomics is to devote more resources to it.

Zwar werden Fehler und Versäumnisse zugestanden, weitreichende Forderungen nach einer etwaigen Restrukturierung des Fachs (etwa durch die stärkere Berücksichtigung heterodoxer Ansätze) werden aber dezidiert zurückgewiesen und sogar als unwissenschaftlich denunziert. ${ }^{20}$

framework on the New Keynesian side together with accumulating empirical difficulties on the Real Business Cycle side eventually drove these two schools into each others arms, albeit in an embrace somewhat lacking in warm affection. The New Keynesians adopted the DSGE framework while the New Classicals borrowed the New Keynesian frictions.“

20 Das beste Beispiel für die faktisch stattfindenden Anpassungsmaßnahmen stellen die kriseninduzierten Modifikationen an DSGE-Modellen dar, die seit wenigen Jahren einen Schwerpunkt makroökonomischer Forschung bilden. DSGE-Modelle basieren auf den oben erläuterten RealBusiness-Cycle-Modellen, enthalten aber zumeist die ebenfalls kurz andiskutierten Neukeynesianischen Friktionen. Von Kritikerseite aufgrund des Fehlens von Finanzmarktkomponenten und der axiomatisch festgeschriebenen Annahme repräsentativer Akteure harsch kritisiert - beides Aspekte, die im Zuge der aktuellen Krise in der Tat maßgeblich waren - und als Hauptbeleg zum Nachweis der Untauglichkeit der Mainstream-Ökonomik herangezogen, wird die Modellklasse seitens des Mainstreams stark verteidigt. So ist der Einbau zuvor absenter Finanzmarktkomponenten (Financial Frictions) gegenwärtig eine Wachstumsbranche (stellvertretend für viele Texte siehe Benchimol, Fourcans 2012). 


\section{Resümee}

Mit Blick auf gesellschaftsweite Großwetterlagen waren die New Classical Macroeconomics Teil einer breiten Bewegung, die zur Durchsetzung eines deutlich marktaffinen Klimas ab Ende der 1970er Jahre beigetragen hat, wodurch insbesondere die Etablierung globaler, weitgehend auf Selbstregulation basierender Finanzmärkte ermöglicht und forciert wurde. „[T]hey offered“, so halten Iversen und Soskice (2006, S. 426) fest, „what we call a depoliticized ,economists“ narrative“ of the rise of restrictive policies“ (vgl. ähnlich auch Chaloupek 2014). Bezogen auf die konkrete Gestaltung wirtschaftspolitischer Institutionen war der Einfluss der New Classical Macroeconomics einerseits limitiert: „[N]ew classical economics had little to say about the causes of institutional design“ (Iversen, Soskice 2006, S. 426). Der Steuerungsskeptizismus dieser Forschungsrichtung war und ist zu radikal, als dass er seitens der gewichtigen wirtschaftspolitischen Organisationen eins zu eins hätte übernommen werden können. Allerdings entstanden im Feld der New Classical Economics wichtige Vorgaben, die dann im Verbund mit den New Keynesian Economics institutionell wirksam wurden, zuvorderst das Dogma politisch unabhängiger Zentralbanken und die Engführung der Zentralbankpolitik auf Geldwertstabilität qua Inflation Targeting. ${ }^{21}$ Es dürfte wesentlich von den weiteren Verlaufsformen und Bearbeitungsmodi der aktuellen Krise abhängig sein, welche Impulse zukünftig von außen auf die Entwicklung des disziplinären Wissens einwirken. Gegenwärtig kann lediglich festgestellt werden, dass die Zentralbankpraktiken in vielen Hinsichten den Empfehlungen aus der Mainstream-Ökonomik konträr gelagert sind (siehe hierzu den instruktiven Text Central Bank-Led Capitalism von Bowman et al. 2013). Der vorliegende Beitrag soll allerdings dafür sensibilisieren, in welch starkem Ausmaß das Feld der Makroökonomik nicht nur durch etablierte disziplinäre Machstrukturen geprägt und in seiner Resonanzfähigkeit affiziert wird (Fourcade et al. 2014), sondern dass auch kognitive Pfadabhängigkeiten in Rechnung gestellt werden müssen. ${ }^{22}$ Es sind solche Pfadabhängigkeiten, die die „Brechungsstärke“ (Bourdieu 1998, S. 19) der Ökonomik als akademischer Disziplin maßgeblich bestimmen.

21 Hierzu Mishkin (1995, S. 8): „The time-consistency perspective provided by the rational expectations revolution has had a major impact in central banking circles. Central banks in many countries have been eager to have their hands tied by having governments impose on them a sole objective of price stability. Indeed, recent legislation in many countries has increased the independence of the central bank and has stipulated price stability as the primary objective for monetary policy“.

22 Mehrling (2006) spricht in einem ähnlichen Sinn von Hysterese. 
Die im zweiten Abschnitt in groben Zügen thematisierte Herausbildung des Keynesianismus der neoklassischen Synthese bildete einen zentralen Bestandteil in der Gesamttransformation der Binnenstrukturen der ökonomischen Disziplin. Morgan und Rutherford (1998) sprechen für die Zeit ab den 1930er Jahren von einem Übergang From Interwar Pluralism to Postwar Neoclassicism. Waren die Wirtschaftswissenschaften bis zu diesem Zeitpunkt durch eine segmentäre Differenzierungsform gekennzeichnet, wie sie bis heute in allen anderen Sparten der Kultur- und Sozialwissenschaften das vorherrschende Muster ist - als ein Neben- und Gegeneinander verschiedener Theorieprogramme und empirischer Forschungsweisen - so kam es im Zuge der Dominanz neoklassisch-gleichgewichtstheoretischer Strömungen zur Herausbildung eines Differenzierungsmodus, der am ehesten als Zentrum-Peripherie-Differenzierung bezeichnet werden kann. Vormals bedeutende Strömungen, etwa der ältere US-amerikanische Institutionalismus, die deutsche historische Schule oder Fortschreibungen klassischer politischer Ökonomie sind zwar in der Folge nicht gänzlich verschwunden, und es sind in der zweiten Hälfte des 20. Jahrhunderts auch neue alternative Spielarten von Ökonomik hinzugekommen (etwa die sogenannten Postkeynesians, die sich der Keynes-Interpretation im Rahmen der neoklassischen Synthese entgegengestellt haben, später die feministische Ökonomik). In der akademischen Disziplin der Ökonomik haben diese Schulen aber nur (noch) prekär-institutionalisierte Nischen- bzw. Randpositionen einnehmen können. Entsprechend hat sich mit der Unterscheidung von Orthodoxie und Heterodoxie in der zweiten Hälfte des 20. Jahrhunderts eine Semantik etabliert, die ihren Ursprung in Hegemoniebestrebungen in religiösen Sphären hat. Für den Zeitraum der letzten ca. 30 Jahre sprechen einige Beobachter der Disziplin von einem Changing Face of Mainstream Economics (Colander et al. 2004), um Auflösungserscheinungen dieses Regimes harscher Zentrum-Peripherie-Differenzierung zum Ausdruck zu bringen. Mit Blick auf viele Forschungsbereiche innerhalb der Wirtschaftswissenschaften gibt es für eine solche Diagnose gute Argumente. Forschungsrichtungen wie den Behavioral Economics oder den Experimental Economics ist in einem relativ kurzen Zeitraum ein Aufstieg von randständigen Spezialmethoden zu weithin akzeptierten Bestandteilen der Curricula gelungen, obgleich dort mit neoklassisch-gleichgewichtstheoretischen Axiomensystemen zumindest in Teilen gebrochen wird. Für die Makroökonomik scheint dies nicht in gleichem Ausmaß zu gelten.

Die im vorliegenden Text abgehandelten Wissensformationen, der Keynesianismus der neoklassischen Synthese, die New Classical Macroeconomics sowie die New Keynesian Economics, bilden einen Varianzraum, der zwar eine erhebliche Binnenkomplexität besitzt und mitunter auch zu sehr unterschiedlich gearteten wirtschaftspolitischen Auffassungen führt, dessen Grundpfeiler sich aber 
vergleichsweise einfach abstecken lassen (beispielsweise entlang der in folgender Tabelle berücksichtigten Dimensionen):

\begin{tabular}{llll}
\hline & Erwartungskonzept & $\begin{array}{l}\text { Markträumung } \\
\text { in der kurzen Frist }\end{array}$ & $\begin{array}{l}\text { Markträumung } \\
\text { in der langen Frist }\end{array}$ \\
\hline $\begin{array}{l}\text { Keynesianismus } \\
\text { der neoklassischen } \\
\text { Synthese }\end{array}$ & adaptiv & nein & ja \\
\hline $\begin{array}{l}\text { New Classical } \\
\text { Macroeconomics }\end{array}$ & rational & ja & ja \\
\hline $\begin{array}{l}\text { New Keynesian } \\
\text { Economics }\end{array}$ & rational & nein & ja \\
\hline
\end{tabular}

Hinzukommt, dass die zeitliche Entwicklung seit den 1970er Jahren eine eher gegenläufige Tendenz zu anderen Forschungsfeldern der Ökonomik erkennen lässt. Zwar kommt es durch den Diskurs der New Keynesian Economics nach der zwischenzeitlichen Hegemonie der New Classical Economics wieder zu einer Auflockerung der „straight jacket of equilibrium“ (Farmer 2012, S. 12), indem vom Paradigma der Markträumung (in der kurzen Frist) abgerückt wird. Zugleich wird aber durch die Übernahme der Theorie rationaler Erwartungen ein anderer Pfeiler (neo-)gleichgewichtstheoretischen Denkens fest im Theoriedesign verankert. Zwar gibt es auch in der Makroökonomik zahlreiche alternative Ansätze, aber die Grenze zwischen Mainstream und Nicht-Mainstream scheint intakter bzw. stabiler als in vielen anderen Forschungsfeldern.

Es wurde im Text ein Eindruck vermittelt, wie stark die moderne MainstreamMakroökonomik im Medium mathematischer Modelle prozessiert und dass dies einen formatierenden Einfluss auf ihre Wissensbestände hat. Backhouse und Laidler (2003, S. 4) haben bereits für die originäre neoklassische Synthese der Mitte des 20. Jahrhunderts festgehalten: „[I]deas that could be fitted into them [mathematisch-modelltheoretische Forschungsrahmen, H.P.] flourished, while those that could not began to seem unimportant“. Auch bei Fourcade (2009, S. 91) wird festgehalten:

Characteristically, all the great revolutions in postwar American economics relied extensively on the success of new formalizing technologies, which made the previous set of rules obsolete and fostered the image of cumulative scientific progres. ${ }^{23}$

23 Dieser Modus wird mittlerweile durch akademische Sozialisations- und Beförderungsmechanismen verstärkt: „By developing these models, graduate students and professors demonstrated 
Hierdurch wurde das im Feld dominante Selbstbewusstsein unterfüttert, wonach die Ökonomik sich - im Unterschied zu anderen Sparten der Kultur- und Sozialwissenschaften - durch irreversiblen und systematischen Wissenszuwachs auszeichne (vgl. zu solchen Whig Histories kritisch Blaug 2001). Im Zuge der Krise $2007 \mathrm{ff}$. haben Kritiker allerdings wiederholt darauf aufmerksam gemacht, dass zahlreiche bereits einmal erreichte Wissensbestände - gerade im Bereich der Geld- und Kredittheorie - im Zuge der immer konsequenteren Umstellung der Makroökonomik auf die Forschungstrajektorien allgemeiner Gleichgewichtstheorie verloren gegangen sind. Turner (2013: 2) spricht pointiert von einer „strange amnesia of modern macroeconomics“. Es geht weit über Anlage und Möglichkeiten des vorliegenden Beitrags hinaus, hierzu Detailuntersuchungen zu liefern (bei Mehrling (1998) findet sich beispielsweise eine Fallstudie zur Transformation der Geldtheorie im Zuge der neoklassischen Synthese und nachfolgender Entwicklungen). ${ }^{24}$ Auch wenn es nicht in den Kompetenzbereich einer Wissenssoziologie der Ökonomik fällt, alternatives ökonomisches Wissen bereitzustellen, kann es eine wichtige Aufgabe sein, Prozesse modellinduzierter kognitiver Pfadabhängigkeiten aufzudecken und die Entwicklung der Wirtschaftswissenschaften auf diese Weise zu problematisieren. Von der Mainstream-Ökonomik kann eine solche Reflexionsleistung vermutlich erst dann erwartet werden, wenn sie den vorherrschenden Umgang mit ihrer eigenen Geschichte überdenkt:

Our profession is agreed, I think, that an economist should have a smattering of economic thought for the same reason that an officer should have decent table manners. But one does not confuse these things with theory and econometrics, or, as the case may be, with tactics and logistics (Leijonhufvud 2006: 2).

that they could jump through hoops; the more hoops they could jump through the higher their income, chances for promotion, and standing in the profession. For many economists economic research has become the art of devising clever models and in doing so demonstrating one's technical virtuosity“ (Colander 1989, S. 33).

24 Ein Auszug: „In building their field anew, postwar authors could not easily proceed inductively from the operational details of individual banks and their interaction in the banking system as prewar authors had done. [...] Instead, postwar authors typically proceeded deductively from the neoclassical theory of value [...]....the theory of value, despite all its problems (e.g., imperfect competition), seemed to them the most solid core of ideas economics had to offer. [...] For better or for worse, postwar monetary theory therefore came to be structured around the ideas of money supply, money demand, and equilibrium“(Mehrling 1998: 293f.). 


\section{Literatur}

Backhouse, Roger (2010): The Puzzle of Modern Economics. Science or Ideology. New York: Cambridge University Press.

Backhouse, Roger E. (2000): Austrian Economics and the Mainstream: View from the Boundary. In: The Quarterly Journal of Austrian Economics 3 (2), S. 31-43.

Backhouse, Roger E. (1997): The Rhetoric and Methodology of Modern Macroeconomics. In: Brian Snowdon, Howard R. Vane und Roger Backhouse (Hg.): Reflections on the Development of Modern Macroeconomics. Cheltenham, UK, Lyme, NH: Edward Elgar, S. 31-54.

Backhouse, Roger E.; Laidler, David (2003): What Was Lost with IS-LM (UWO Department of Economics Working Papers. The University of Western Ontario).

Bank of England (2009): The Role of Macroprudential Policy. A Discussion Paper. Online verfügbar unter http://www.bankofengland.co.uk/publications/Documents/other/financialst ability/roleofmacroprudentialpolicy091121.pdf.

Benchimol, Jonathan; Fourcans, Andre (2012): Money and Risk in a DSGE Framework: A Bayesian Application to the Eurozone. In: Journal of Macroeconomics 34, S. 95-111.

Bernanke, Ben S. (2004): The Great Moderation. Remarks by Governor Ben S. Bernanke. At the Meetings of the Eastern Economic Association, Washington, DC. Online verfügbar unter http://www.federalreserve.gov/Boarddocs/Speeches/2004/20040220/.

Bernanke, Ben S.; Mishkin, Frederic S. (1997): Inflation Targeting: A New Framework For Monetary Policy? (NBER Working Paper Series, 5893). Online verfügbar unter http://www.nber.org/ papers/w5893.pdf.

Blaug, Mark (2001): No History of Ideas, Please, We're Economists. In: Journal of Economic Perspectives 15 (1), S. 145-164.

Blinder, Alan S. (1997): The Fall and Rise von Keynesian Economics. In: Brian Snowdon und Howard R. Vane (Hg.): A Macroeconomics Reader. London: Routledge, S. 109-134.

Bourdieu, Pierre (1998): Vom Gebrauch der Wissenschaft. Für eine klinische Soziologie des wissenschaftlichen Feldes. Konstanz: UVK.

Bowman, Andrew; Erturk, Ismael; Froud, Julie; Johal, Sukhdev; Leaver, Adam; Moran, Michael; Williams, Karel (2013): Central Bank-Led Capitalism? In: Seattle Law Review 36 (2), S. 455-487.

Braun, Benjamin (2014): Why Models Matter: The Making and Unmaking of Governability in Macroeconomic Discourse. In: Journal of Critical Globalisation Studies (7), S. 48-79.

Brzoza-Brzezina, Michal; Kolada, Marcin; Makarski, Krzysztof (2011): The Anatomy of Standard DSGE Models with Financial Frictions (National Bank of Poland Working Paper, 80). Online verfügbar unter http://www.nbp.pl/publikacje/materialy_i_studia/80_en.pdf.

Caballero, R. J. (2010): Macroeconomics after the Crisis: Time to Deal with the Pretense-ofKnowledge Syndrome. In: Journal of Economic Perspectives 24, S. 85-102.

Caspari, Volker; Schefold, Bertram (Hg.) (2011): Wohin steuert die ökonomische Wissenschaft? Ein Methodenstreit in der Volkswirtschaftslehre. Frankfurt am Main: Campus.

Chaloupek, Günther (2014): Alternative theoretische Ansätze und die Qualität des wirtschaftspolitischen Diskurses. In: Katrin Hirte, Sebastian Thieme und Walter Otto Ötsch (Hg.): Wissen! Welches Wissen? Zu Wahrheit, Theorien und Glauben sowie ökonomischen Theorien. Marburg: Metropolis, S. 303-318.

Chari, Varadarajan (2010): Testimony before the Committee on Science and Technology, Subcommittee on Investigations and Oversight, U.S. House of Representatives. Online verfügbar unter http://people.virginia.edu/ ey2d/Chari_Testimony.pdf. 
Chari, Varadarajan (1999): Nobel Laureate Robert E. Lucas, Jr.: Architect of Modern Macroeconomics. In: Quarterly Review, Federal Reserve Bank of Minneapolis 23 (2), S. 2-11.

Clarida, Richard; Gali, Jordi; Gertler, Mark (1999): The Science of Monetary Policy: A New Keynesian Perspective. In: Journal of Economic Literature XXXVII (December), S. 1661-1707. Online verfügbar unter http://www.nyu.edu/econ/user/gertlerm/science.pdf.

Coddington, Alan (1976): Keynesian Economics. The Search for First Principles. In: Journal of Economic Literature 14, S. 1258-1273.

Colander, David; Holt, Richard P. F. Rosser J. Barkley (2004): The Changing Face of Mainstream Economics. In: Review of Political Economy 16 (4), S. 485-499.

Colander, David (2002): Functional Finance, New Classical Economics and Great Great Grandsons (Middlebury College Economics Discussion Paper, 02-34).

Dixon, Huw D. (1997): The Role of Imperfect Competition in New Keynesian Economics. In: Brian Snowdon, Howard R. Vane und Roger Backhouse (Hg.): Reflections on the Development of Modern Macroeconomics. Cheltenham, UK, Lyme, NH: Edward Elgar, S. 158-203.

Eicker-Wolf, Kai (2003): Vom hydraulischen Keynesianismus zur radikalen politischen Ökonomie (RPÖ). Keynesianische wirtschaftspolitische Konzeptionen in der Bundesrepublik Deutschland nach dem Scheitern der Globalsteuerung. Marburg: Metropolis.

Evans, George W.; Ramey, Garey (2001): Adaptive Expectations, Underparameterization and the Lucas Critique (University of California, San Diego, Discussion Paper, 2001-11).

Farmer, J. Doyne (2012): Economics Needs to Treat the Economy as a Complex System (Paper for the INET Conference "Rethinking Economics and Politics", April 14, 2012). Online verfügbar unter http://ineteconomics.org/sites/inet.civicactions.net/files/farmer_berlinpaper. pdf.

Farmer, J. Doyne; Foley, Duncan (2009): The Economy Needs Agent-Based Modelling. In: Nature 460 (7256), S. 685-686. DOI: 10.1038/460685a.

Fourcade, Marion (2009): Economists and Societies. Discipline and Profession in the United States, Britain, and France, 1890s to 1990s. Princeton, NJ: Princeton Univ. Press.

Fourcade, Marion; Ollion, Etienne; Algan, Yann (2014): The Superiority of Economists (MaxPo Discussion Paper, 14/3). Online verfügbar unter http://www.maxpo.eu/pub/maxpo_dp/ maxpodp14-3.pdf.

Galí, Jordi (2008): Monetary Policy, Inflation, and the Business Cycle. An Introduction to the New Keynesian Framework. Princeton, N.J: Princeton University Press.

Goodfriend, Marvin (2007): How the World Achieved Consensus on Monetary Policy. Cambridge, Mass. (NBER Working Paper Series, 13580). Online verfügbar unter http://www.nber.org/ papers/w13580.

Gordon, Robert J. (1990): What Is New-Keynesian Economics? In: Journal of Economic Literature 28 (3), S. $1115-1171$.

Grauwe, Paul de (2010): Top-Down versus Bottom-Up Macroeconomics. In: CESifo Economic Studies 56 (4), S. 465-497.

Greenwald, Bruce; Stiglitz, Joseph E. (1987): Keynesian, New Keynesian, and New Classical Economics (NBER Working Paper Series, 2160).

Hoover, Kevin D. (1992): The Rational Expectations Revolution: An Assessment. In: Cato Journal 12 (1), S. 81-106.

Hoover, Kevin D. (1988): The New Classical Macroeconomics. A Sceptical Inquiry. Oxford, UK, New York, NY, USA: B. Blackwell.

Hoover, Kevin D. (1984): Two Types of Monetarism. In: Journal of Economic Literature XXII (March), S. 58-76. 
Hoover, Kevin D.; Young, Warren (2011): Rational Expectations: Retrospect and Prospect. A Panel Discussion with Michael Lovell, Robert Lucas, Dale Mortensen, Robert Shiller, Neil Wallace. Moderated by Kevin Hoover and Warren Young. (CHOPE Working Paper, 2011-10). Online verfügbar unter http://www.dklevine.com/archive/refs4786969000000000227.pdf.

Howitt, Peter (2006): Monetary Policy and the Limitations of Economic Knowledge. In: David C. Colander (Hg.): Post Walrasian Macroeconomics. Beyond the Dynamic Stochastic General Equilibrium Model. Cambridge, New York: Cambridge University Press, S. 347-367.

Iversen, Torben; Soskice, David (2006): New Macroeconomics and Political Science. In: Annual Review of Political Science 9 (1), S. 425-453.

Jonas, Friedrich (1964): Das Selbstverständnis der ökonomischen Theorie. Berlin: Duncker \& Humblot.

Keynes, J. M. (1936): The General Theory of Employment, Interest and Money. London: Macmillan.

Keynes, John M. (1924): A Tract on Monetary Reform. London: Macmillan.

Klamer, Arjo (1984): Conversations with Economists. New Classical Economists and Opponents speak out on the Current Controversy in Macroeconomics. Totowa, N.J: Rowman \& Allanheld.

Klein, Lawrence R. (1966): The Keynesian Revolution. New York: Macmillan.

Kuhn, Thomas S. (1962): The Structure of Scientific Revolutions. Chicago: Univ. of Chicago Press. Kydland, Finn E.; Prescott, Edward C. (1982): Time to Build and Aggregate Fluctuations. In: Econometrica 50 (6), S. 1345-1370.

Laidler, David E. W (1999): Fabricating the Keynesian Revolution. Studies of the Inter-War Literature on Money, the Cycle, and Unemployment. Cambridge [England], New York: Cambridge University Press.

Lebaron, Frédéric (2000): La croyance économique. Les économistes entre science et politique. Paris: Seuil.

Leijonhufvud, Axel (2009): Macroeconomics and the Crisis: A Personal Appraisal (ARC 2009 Opening Lecture, Crisis and Reform, Brussels). Online verfügbar unter http://ec.europa.eu/ economy_finance/events/2009/20091015/0-leijonhufvud_en.pdf.

Leijonhufvud, Axel (2006): The Uses of the Past (Università degli Studi di Trento, Dipartimento di Economia, Discussion Paper, 3/2006). Online verfügbar unter http://web.unitn.it/files/ 3_06_leijonhufvud.pdf.

Leijonhufvud, Axel (1968): On Keynesian Economics and the Economics of Keynes. A Study in Monetary Theory. New York [etc.]: Oxford University Press.

Lucas, Robert E. [JR.] (2003): Macroeconomic Priorities. In: American Economic Review 93 (1), S. 1-14.

Lucas, Robert E. (1988): On the Mechanics of Economic Development. In: Journal of Monetary Economics 22, S. 3-42.

Lucas, Robert E. (1977): Understanding Business Cycles. In: Journal of Monetary Economics 5 (Supplement, Carnegie Rochester Conference Series on Public Policy), S. 7-29.

Lucas, Robert E. (1976): Econometric Policy Evaluation: A Critique. In: Karl Brunner und Allan H. Meltzer (Hg.): Carnegie-Rochester Conference Series. Vol. 1. Amsterdam: North Holland, S. 19-46.

Lucas, Robert E. (1972): Expectations and the Neutrality of Money. In: Journal of Economic Theory (4), S. 103-124.

Lucas, Robert E.; Sargent, Thomas J. (1979): After Keynesian Macroeconomics. In: Federal Reserve Bank of Minneapolis Quarterly Review 3 (2), S. 1-16. 
MacKenzie, Donald (2008): An Engine, not a Camera. How Financial Models Shape Markets. First Paperback Edition. Cambridge, London: MIT Press.

Maesse, Jens (Hg.) (2013): Ökonomie, Diskurs, Regierung. Interdisziplinäre Perspektiven. Wiesbaden: Springer Fachmedien Wiesbaden; Imprint: Springer VS.

Mankiw, Nicholas Gregory; Romer, David (1991): New Keynesian Economics. Volume 1. Imperfect Competition and Sticky Prices. Cambridge, Mass. u.a: MIT Pr.

Mehrling, Perry (2011): The new Lombard Street. How the Fed became the Dealer of Last Resort. Princeton, N.J: Princeton University Press.

Mehrling, Perry (2006): The Evolution of Macroeconomics: The origins of Post Walrasian Macroeconomics. In: David C. Colander (Hg.): Beyond Microfoundations. Post Walrasian Macroeconomics. Cambridge: Cambridge University Press, S. 71-86.

Mehrling, Perry (1998): The Money Muddle: The Transformation of American Monetary Thought, 1920-1970. In: History of Political Economy 30 (Supplement), S. 293-396.

Michaelis, Jochen (2013): Und dann werfen wir den Computer an - Anmerkungen zur Methodik der DSGE-Modelle (MAGKS. Joint Discussion Paper Series in Economics, 23-2013). Online verfügbar unter http://www.uni-marburg.de/fb02/makro/forschung/magkspapers/23-201 3_michaelis.pdf.

Mirowski, Philip (1999): More Heat Than Light. Economics as Social Physics, Physics as Nature's Economics. Cambridge: Cambridge Univ. Press.

Mishkin, Frederic S. (1995): The Rational Expectations Revolution: A Review Article of: Preston J. Miller, Ed.: The Rational Expectations Revolution, Readings from the Front Line (NBER Working Paper Series, 5043). Online verfügbar unter http://www.nber.org/papers/w5043.pdf.

Mitchell, Timothy (1998): Fixing the Economy. In: Cultural Studies 12 (1), S. 82-101.

Morgan, Mary. S.; Rutherford, Malcolm (Hg.) (1998): From Interwar Pluralism to Postwar Neoclassicism (1998): Durham, NC: Duke Univ. Press.

Muth, John F. (1961): Rational Expectations and the Theory of Price Movements. In: Econometrica 29 (3), S. 315-335.

Pearce, Kerry A.; Hoover, Kevin D. (1995): After the Revolution: Paul Samuelson and the Textbook Keynesian Model. In: History of Political Economy 27 (Supplement), S. 183-216.

Phillips, A.W.H. (1958): The Relationship between Unemployment and the Rate of Change of Money Wages in the United Kingdom 1861-1957. In: Economica 25 (100), S. 283-299.

Piketty, Thomas (2014): Capital in the Twenty-First Century. Cambridge, Mass, London: The Belknap Press of Harvard University Press.

Prescott, Edward C. (1986): Theory Ahead of Business Cycle Measurement. Federal Reserve Bank of Minneapolis (Quarterly Review, Vol. 10, Nr. 4).

Sargent, Thomas J. (2010): Interview with Thomas Sargent. The Federal Reserve Bank of Minneapolis. Minneapolis. Online verfügbar unter http://www.minneapolisfed.org/publications_ papers/pub_display.cfm?id=4526.

Schlefer, Jonathan (2012): The assumptions economists make. Cambridge, Mass.: Belknap Press of Harvard University Press.

Schumpeter, Joseph A. (2009): Geschichte der ökonomischen Analyse. Göttingen: Vandenhoeck \& Ruprecht.

Snowdon, Brian; Vane, Howard R. (2005): Modern Macroeconomics. Its Origins, Development and Current State. Cheltenham, UK, Northhampton, MA: E. Elgar.

Snowdon, Brian; Vane, Howard R. (1997): To Stabilize or not to Stabilize: Is that the Question? In: Brian Snowdon, Howard R. Vane und Roger Backhouse (Hg.): Reflections on the Development of Modern Macroeconomics. Cheltenham, UK, Lyme, NH: Edward Elgar, S. 1-30. 
Solow, Robert M. (1956): A Contribution to the Theory of Economic Growth. In: The Quarterly Journal of Economics 70 (1), S. 65-94.

Togati, Teodoro Dario (1998): Keynes and the Neoclassical Synthesis. Einsteinian versus Newtonian Macroeconomics. London, New York: Routledge (21).

Turner, Adair (2013): Credit, Money and Leverage: What Wicksell, Hayek and Fisher Knew and Modern Macroeconomics Forgot (Conference Paper, Stockholm School of Economics, Towards a Sustainable Financial System). Online verfügbar unter http://www.princeton.edu/ jrc/events_archive/repository/credit_money_turner/Credit_Money_Leverage.pdf.

Vroey, Michel de; Hoover, Kevin D. (2004): Introduction: Seven Decades of the IS-LM Model. In: History of Political Economy 36 (Annual Supplement), S. 1-11.

Weintraub, Eliot Roy (2002): How Economics became a Mathematical Science. Durham: Duke Univ. Press.

Weintraub, E. Roy (1986): Microfoundations. The Compatibility of Microeconomics and Macroeconomics. Cambridge: Cambridge Univ. Press.

Woodford, Michael (2009): Convergence in Macroeconomics: Elements of the New Synthesis. In: American Economic Journal: Macroeconomics 1 (1), S. 267-279. 